\title{
Vertical Augmentation of Maxillary Posterior Alveolar Ridge Using Allogenic Block Bone Graft and Simultaneous Maxillary Sinus Graft
}

\author{
Eun-Young Lee, Eun-Suk Kim ${ }^{1}$, Kyoung-Won Kim \\ Department of Oral and Maxillofacial Surgery, Medical Research Institute, College of Medicine, Chungbuk National University, \\ ${ }^{1}$ Department of Oral and Maxillofacial Surgery, Jukjeon Dental Hospital, College of Dentistry, Dankook University
}

\begin{abstract}
The maxillary posterior area is the most challenging site for the dental implant. Although the sinus graft is a predictable and successful technique for rehabilitation of atrophic and pneumatized posterior maxilla, when there is severe destruction of alveolar bone, a very long crown length remains challenging after successful dental implants installation with sinus graft. We performed vertical augmentation of the maxillary posterior alveolar ridge using the allogenic block bone graft with a simultaneous sinus graft using allogenic and heterogenic bone chips. After about six months, we installed the dental implant. After this procedure, we achieved a more favorable crown-implant fixture ratio and better results clinically and biomechanically. This is a preliminary report of vertical augmentation of maxillary posterior alveolar ridge using allogenic block bone graft and simultaneous maxillary sinus graft. Further research requires longer observation and more patients.
\end{abstract}

Key words: Maxillary ridge augmentation, Sinus floor augmentations

\section{Introduction}

Dental implant installation requires a quality and quantity of alveolar bone sufficient to support implantation. A severely resorbed maxillary posterior area presents many limitations for a conventional implant installation. Such limitations are usually due to the atrophic alveolar bone after loss of teeth, poor bone quality, and maxillary sinus pneumatization[1,2]. Therefore, different techniques have been developed over the last several decades. The maxillary sinus graft technique is widely used for rehabilitation of the edentulous posterior molar area of maxilla and is deemed a most predictable procedure[3].

Maxillary sinus pneumatization progresses more severely after teeth loss, and the bone quality of the edentulous molar region worsens from lack of functional load. There are two aspects in the limitations and difficulties of maxillary posterior molar area: the downward direction of sinus floor due to the maxillary sinus pneumatization, and the upward direction of alveolar bone loss due to destruction and resorption of the alveolar bone with chronic periodontitis. In general, the vertical augmentation of the atrophic alveolar

RECEIVED August 7, 2014, REVISED August 28, 2014, ACCEPTED September 22, 2014

Correspondence to Kyoung-Won Kim

Department of Oral and Maxillofacial Surgery, Medical Research Institute, Chungbuk National University College of Medicine

52 Naesudong-ro, Seowon-gu, Cheongju 362-763, Korea

Tel: 82-43-269-6383, Fax: 82-43-269-6387, E-mail: kwkim@chungbuk.ac.kr

Copyright (C) 2014 by The Korean Association of Maxillofacial Plastic and Reconstructive Surgeons. All rights reserved.

(c) This is an open access article distributed under the terms of the Creative Commons Attribution Non-Commercial License (http://creativecommons. org/licenses/ by-nc/3.0) which permits unrestricted non-commercial use, distribution, and reproduction in any medium, provided the original work is properly cited. 
bone is a more difficult procedure than the maxillary sinus graft procedure. A main cause of alveolar bone height deficiency is maxillary sinus pneumatization or alveolar bone destruction, so evaluation of residual alveolar bone height in the maxillary posterior region is crucial.

We reported on the vertical augmentation of maxillary posterior alveolar ridge using mandibular ramal block bone graft and sinus graft[4]. We thought that the autogenic block bone graft was a good choice for vertical ridge augmentation. However, an autogenic block bone graft is disadvantageous as there is additional surgery and longer operation time for taking autogenic block bone, donor site morbidity of discomfort, pain and swelling, and limited amount of autogenic block bone. Thus we used an allogenic block bone graft for vertical augmentation instead of an autogenic block bone graft.

\section{Case Report}

1. Preoperative evaluation of the alveolar bone and maxillary sinus cavity

A 46-year-old man was referred from local clinic for the dental implant and bone graft. For the initial evaluation we took a panoramagraphy. This showed a very thin remaining alveolar ridge after tooth loss (\#26) with severe alveolar bone destruction due to periodontal problem and slight pneumatization of maxillary sinus cavity. For the precise evaluation of the residual alveolar bone and left maxillary sinus cavity, we took a dental computed tomography (CT) scan. The CT scan confirmed the panorama- graphic finding of severe alveolar bone damage, especially the buccal area, resulting from the periodontitis at the maxillary left first molar, \#26. The residual alveolar ridge was very thin with mild pneumatization of the left maxillary sinus cavity (Fig. 1).

\section{Vestibular incision for the recipient site preparation} for vertical augmentation of maxillary alveolar ridge and lateral window formation for sinus bone graft

We did a vestibular incision on the movable mucosal area horizontally and parallel with a residual alveolar ridge, instead of a crestal incision, for the simultaneous installation of the dental implant with sinus graft. We did a careful dissection of the mucoperiosteal flap elevation under the sinus floor to prepare the recipient site for vertical augmentation of maxillary alveolar ridge. The mucoperiosteal flap elevation extended to the palatal side to allow room for vertical augmentation without damage or perforation of the flap. We then made a lateral window opening with a round bur using ordinary methods and created a lateral bony window for sinus graft. The maxillary sinus membrane was elevated for the maxillary sinus graft. An allogenic block bone was hydrated sufficiently with normal saline (Fig. 2).

3. Vertical augmentation of maxillary posterior alveolar ridge with a allogenic block bone and simultaneous maxillary sinus graft

After evaluation of the recipient site shape and size for vertical augmentation of the maxillary posterior alveolar
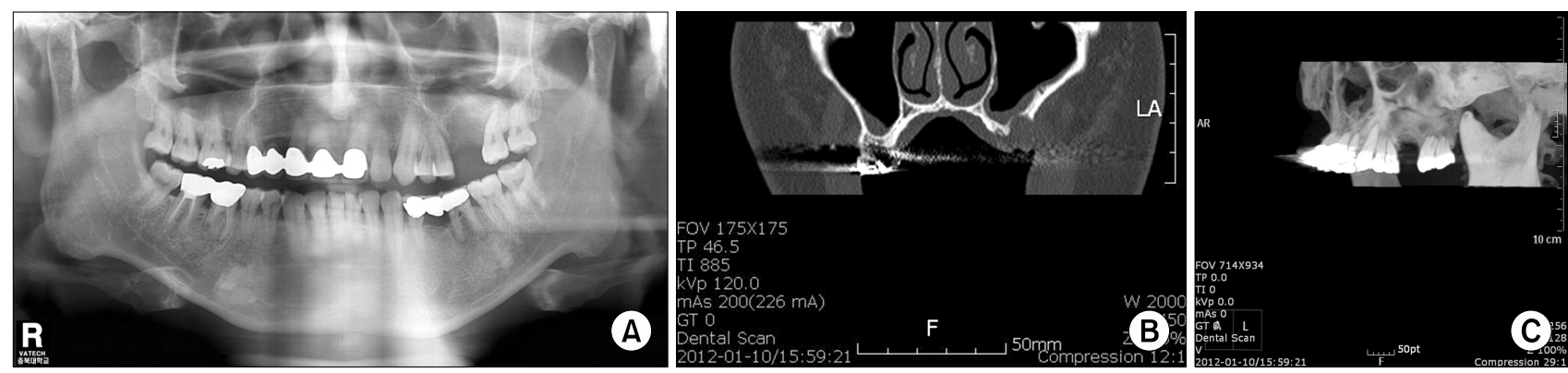

Fig. 1. Diagnosis \& radiographic evaluation of the remaining alveolar ridge, maxillary sinus cavity and mandibular ramus. (A) Panoramagraphy of the patient shows a very thin remaining alveolar ridge after tooth loss (\#26) with severe alveolar bone destruction due to periodontal problem and pneumatization of maxillary sinus cavity. (B) The coronal view of dental computed tomography (CT) scan shows a very thin remaining alveolar ridge (height; about $1 \mathrm{~mm}$ ) and severe buccal alveolar bone destruction due to periodontal problem with slight mucosal thickening in left maxillary sinus cavity. (C) The 3-dimensional view of dental CT scan shows buccal alveolar bone destruction due to periodontal problem between maxillary right 2 nd premolar and 2 nd molar area. 

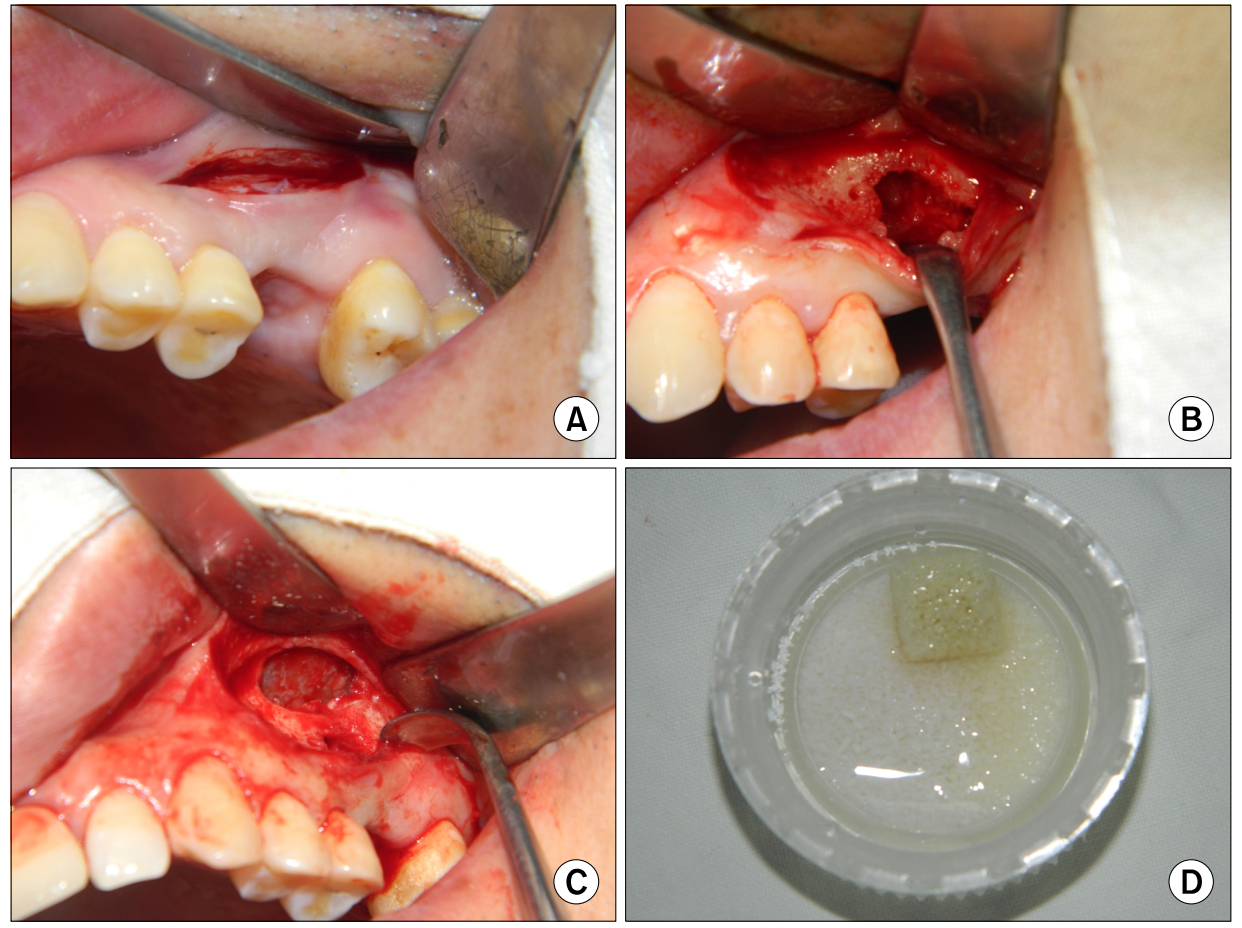

Fig. 2. Vestibular incision for the vertical alveolar ridge augmentation and simultaneous maxillary sinus graft. (A) Vestibular horizontal incision parallel with residual alveolar bone on the movable mucosal area was made. (B) Careful elevation of the mucoperiosteal flap with the periosteal elevator shows buccal alveolar bone destruction area under maxillary sinus floor and makes space for the allogenic block bone graft. (C) Making lateral bony window using \#6 round bur for the maxillary sinus graft and removal of bony lateral window with freer periosteal elevator. (D) The allogenic block bone hydration with normal saline.
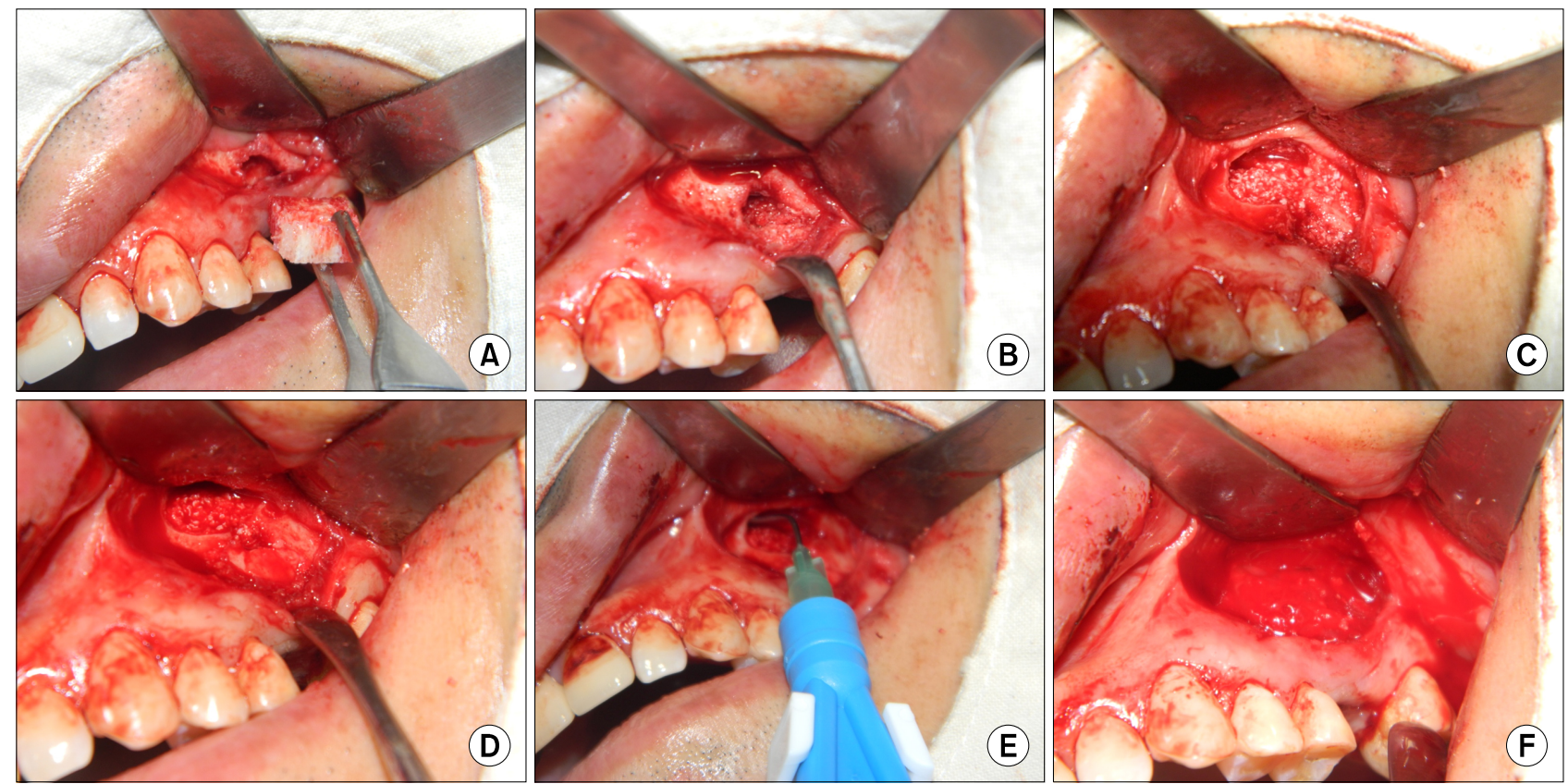

Fig. 3. Vertical alveolar ridge augmentation with the allogenic block bone graft and simultaneous maxillary sinus graft. (A) Application of the allogenic block bone into the prepared mucoperiosteal pocket for trimming of the allogenic block bone according to the recipient site dimension and shape. (B) Insertion of the allogenic block bone into the prepared mucoperiosteal pocket under the maxillary sinus floor for the vertical augmentation. (C) Maxillary sinus graft with layered graft using allogenic bone chips and heterogenic bone chips in the maxillary sinus cavity. (D) Application of the lateral bony window (cortical bone) on the buccal side of the allogenic block bone graft. (E) Application of fibrin sealant (Beriplast ${ }^{\circledR}$; Behring $\mathrm{GmbH}$, Marburg, Germany) for the fixation of the sinus graft and vertical augmentation materials. (F) After initial setting of the fibrin sealant. 
ridge, we trimmed the allogenic block bone with a bur and bone rongeur following the shape and size of recipient site. We put the trimmed allogenic block into the recipient site, taking care not to damage or perforate the mucoperiosteal flap. The preformed recipient site, shaped into a pocket, was surrounded by thick keratinized attached gingival and palatal mucosa. The allogenic block bone was put into the recipient site surrounded by a thick mucoperiosteal flap as a wedge, so there was no need for fixation with screws. After the allogenic block bone graft, small allogenic bone chips and heterogenic bone chips were put in a dead space between the allogenic block bone and overlying mucoperiosteal flap. Simultaneous maxillary sinus graft was performed with allogenic bone chips and heterogenic bone chips by the layered graft technique described in our previous report[5]. After sinus graft, the lateral bony window was opened on the lateral side of the allogenic block bone graft area. We wanted the lateral bony window rolled as a cortical autogenous bone wall for maintenance of volume and a more favorable result of vertical augmentation. We applied a tissue adhesive (fibrin sealant, Beriplast ${ }^{\circledR}$; Behring $\mathrm{GmbH}$, Marburg, Germany) to fix the grafted materials in the sinus cavity and vertical augmentation area. We closed the wound with 3-0 black silk. After operation, we took a panoramagraphy to evaluate the results (Fig. 3).

\section{Dental implant installation}

After six months, we installed a dental implant on the previous vertical augmentation of maxillary posterior alveolar ridge and sinus graft site. During drilling for placement of a dental implant, we took a specimen from the allogenic block bone graft area. We made slides (H\&E stain and M\&T stain) for light microscopic evaluation of new bone formation on the allogenic block bone graft site. These slides show new bone formation beside a slightly resorbed allogenic block bone particle for remodeling. We drilled very carefully to prevent separating the allogenic block
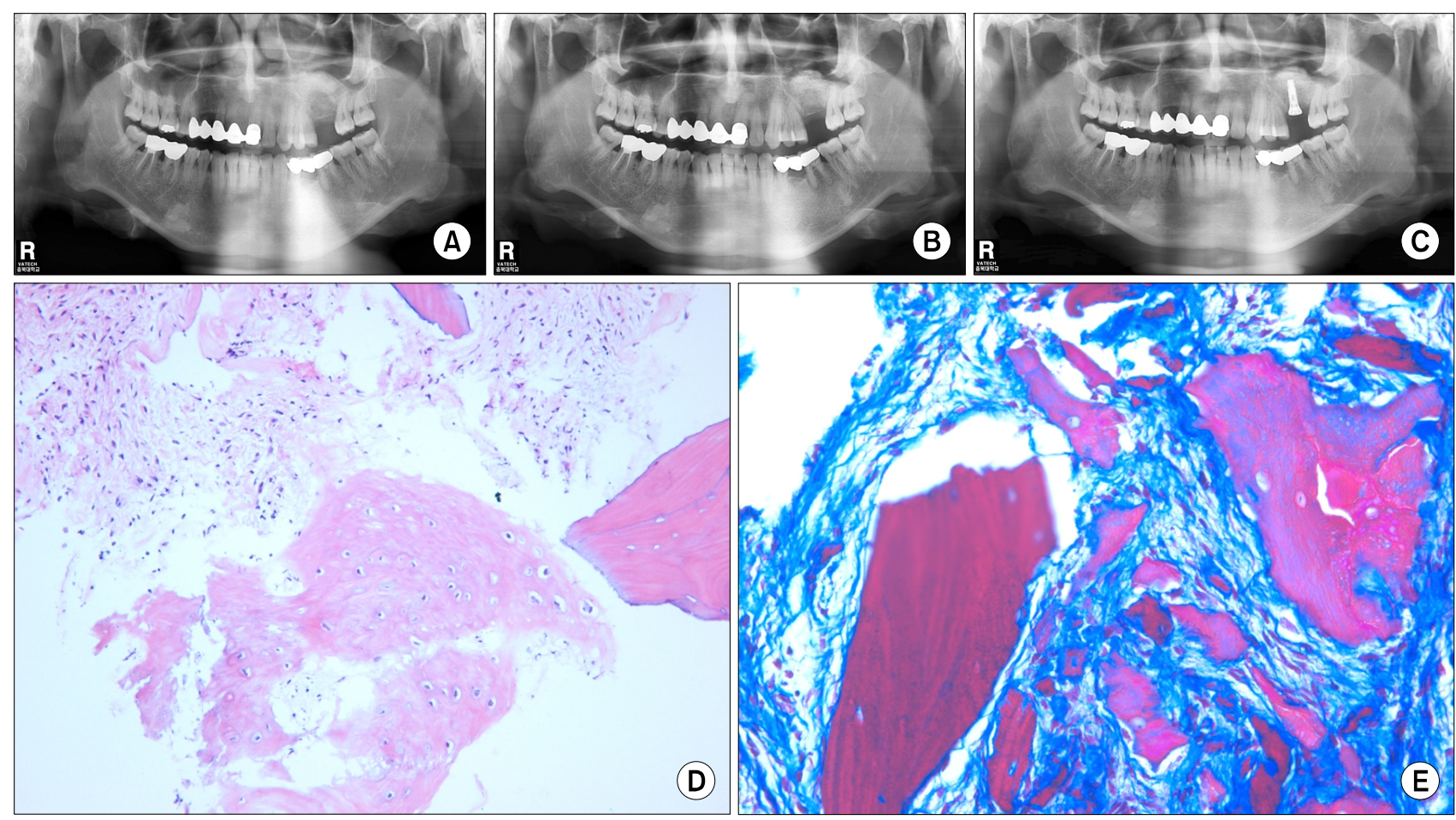

Fig. 4. Dental implant installation on the previous vertical augmentation using allogenic bone graft and sinus graft site. (A) Panoramagraphy after vertical alveolar ridge augmentation using allogenic block bone graft and simultaneous maxillary sinus graft (immediate postoperative). (B) Panoramagraphy on postoperative 5 months shows a good healing process. (C) Installation of the dental implant on the vertical ridge augmentation and sinus graft site after 5 months. (D) This slide was made from a specimen during drilling for a dental implant. It shows new bone formation (low center) beside an allogenic block bone particle with slight resorption (right side) $(\mathrm{H} \& \mathrm{E}, \times 200)$. (E) This slide shows new bone formation with a resorbed allogenic block bone particle (upper right) (M\&T stain, $\times 200$ ). 
bone and host maxillary alveolar ridge (Fig. 4).

\section{Fabrication of the final prosthodontic appliance}

We made a final prosthodontic appliance on the dental implant fixture after five months. We expect a good prognosis of the final prosthodontic appliance, because the crown to implant fixture ratio was good due to vertical augmentation of maxillary posterior alveolar ridge (Fig. 5).

\section{Discussion}

The maxillary posterior molar area presents challenges of bone quantity and quality. After loss of teeth, alveolar bone resorption of maxillary posterior area is more rapid than other sites and maxillary sinus pneumatization progresses more rapidly. Crestal bone resorption, combined with the pneumatization of the maxillary sinus that occurs after posterior teeth loss, often results in inadequate bone volume for endosseous dental implant placement[6,7]. When residual alveolar bone is very thin, a main cause of deficiency of alveolar bone height is from pneumatization of the maxillary sinus cavity or destruction of alveolar bone. When there is crestal alveolar bone resorption or destruction, the clinical crown length of final prosthodontics is too long despite successful maxillary sinus graft procedures. This can cause poor crown to implant fixture ratio and poor clinical and biomechanical prosthodontics prognosis.

Aghaloo and Moy[3] wrote a systematic review of a main online database and a manual search of relevant articles between 1980 and 2005 from referred journals. They found

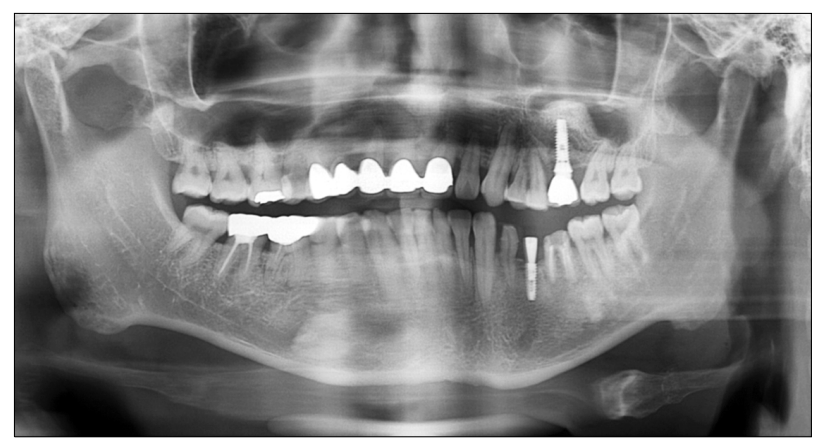

Fig. 5. Twenty months follow-up panoramagraphy after loading ( 2 years and 6 months after bone graft) of final prosthodontic treatment shows favorable crown-implant ratio and good healing state of vertical augmentation and sinus graft. good documentation of the maxillary sinus augmentation procedure. From this review, the long-term clinical success and survival ( $>5$ years) of implants placed into augmented bone, regardless of graft materials used, appears to be similar to or better than that of implants placed using conventional protocol with no grafting procedure. Therefore the maxillary sinus graft technique is widely used for rehabilitation of the edentulous posterior molar area of maxilla and is accepted as a predictable procedure[3,8,9].

However, vertical augmentation of the alveolar ridge is very difficult. There are numerous procedures reported, including guided bone regeneration with membrane, onlay or veneer graft, combinations of onlay, veneer, interpositional inlay graft, distraction osteogenesis. Autogenous block bone graft might be first choice for vertical augmentation of maxillary posterior alveolar ridge. We reported on vertical augmentation of maxillary posterior alveolar ridge using mandibular ramal block bone graft and sinus graft[4]. We concluded that the autogenic block bone graft for the vertical ridge augmentation gave good results. In this study, we used an allogenic block bone instead of an autogenic block bone for vertical augmentation of maxillary posterior alveolar ridge. The main advantages of the allogenic block bone graft are no additional surgical procedures and no donor site morbidity. During vertical augmentation procedures, an allogenic block bone was easier to trim and shape than autogenic mandibular ramal block bone. The allogenic block bone was placed as a wedge in a preformed recipient site like a pocket under the mucoperiosteal flap. There was no need for fixation with screws, because the recipient site was surrounded by thick keratinized attached gingiva and palatal mucosa. After insertion of the allogenic block bone, remaining gap between mucoperiosteal flap and allogenic block bone was filled with allogenic bone chips and heterogenic bone chips without dead space. Simultaneous maxillary sinus graft was performed with allogenic bone chips and heterogenic bone chips as a layered graft technique. We reported that the lower layer using allogenic graft materials gave good osteoinduction and osteoconduction from the maxillary sinus cavity wall, and heterogenic graft materials for the upper layer prevents rapid resorption from maxillary sinus pneumatization[5]. After sinus graft, we placed a lateral bony window on the lateral side of the allogenic block bone graft area. We 
wanted that the lateral bony window rolled as a cortical autogenous bone wall for maintenance of volume during healing and for prevention of rapid resorption of allogenic block bone graft. That might be for more favorable results in vertical augmentation. Finally we fixed grafted materials with a tissue adhesive (fibrin sealant, Beriplast ${ }^{\circledR}$; Behring $\mathrm{GmbH}$ ). A tissue adhesive has many advantages[10] and we reported about fibrin sealant for management of sinus membrane perforation during maxillary sinus bone graft[11]. After six months, we observed relatively more resorption of allogenic block bone than autogenic mandibular ramal block bone. It might be a limitation of an allogenic block bone.

We did vertical augmentation of maxillary posterior alveolar ridge using an allogenic block bone graft and simultaneous maxillary sinus graft. Six months later, we installed a dental implant. A final prosthodontic appliance was placed after five months. The final prosthodontics had a better crown to implant fixture ratio and a result that is more satisfactory clinically and biomechanically. We think the allogenic block bone is an option for vertical augmentation instead of autogenic block bone. However, this study is a case report; more cases with longer observation are needed.

\section{Acknowledgements}

This work was supported by a 2012 research grant from Chungbuk National University.

\section{References}

1. Lorenzetti M, Mozzati M, Campanino PP, Valente G. Bone augmentation of the inferior floor of the maxillary sinus with autogenous bone or composite bone grafts: a histologic-histomorphometric preliminary report. Int $\mathrm{J}$ Oral Maxillofac Implants 1998;13:69-76.

2. Barone A, Santini S, Sbordone L, Crespi R, Covani U. A clinical study of the outcomes and complications associated with maxillary sinus augmentation. Int $\mathrm{J}$ Oral Maxillofac Implants 2006;21:81-5.

3. Aghaloo TL, Moy PK. Which hard tissue augmentation techniques are the most successful in furnishing bony support for implant placement? Int J Oral Maxillofac Implants 2007; 22 Suppl:49-70.

4. Kim KW, Lee EY. Inus graft and vertical augmentation of maxillary posterior alveolar ridge using mandibular ramal block bone graft. J Korean Assoc Maxillofac Plast Reconstr Surg 2010;32:276-81.

5. Kim KW, Lee EY. Layered graft technique with allogenic and heterogenic graft materials in maxillary sinus graft. Chungbuk Med J 2013;23:115-23.

6. John HD, Wenz B. Histomorphometric analysis of natural bone mineral for maxillary sinus augmentation. Int J Oral Maxillofac Implants 2004;19:199-207.

7. McDermott NE, Chuang SK, Woo VV, Dodson TB. Maxillary sinus augmentation as a risk factor for implant failure. Int $\mathrm{J}$ Oral Maxillofac Implants 2006;21:366-74.

8. Sorní M, Guarinós J, García O, Peñarrocha M. Implant rehabilitation of the atrophic upper jaw: a review of the literature since 1999. Med Oral Patol Oral Cir Bucal 2005;10 Suppl 1:E45-56.

9. Jensen T, Schou S, Stavropoulos A, Terheyden H, Holmstrup P. Maxillary sinus floor augmentation with Bio-Oss or Bio-Oss mixed with autogenous bone as graft: a systematic review. Clin Oral Implants Res 2012;23:263-73.

10. Hallman M, Nordin T. Sinus floor augmentation with bovine hydroxyapatite mixed with fibrin glue and later placement of nonsubmerged implants: a retrospective study in 50 patients. Int J Oral Maxillofac Implants 2004;19:222-7.

11. Kim KW, Lee EY. Management of sinus membrane perforation during maxillay sinus bone graft. Chungbuk Med J 2011;21:195-205. 